\title{
Stable sets in one-seller assignment games
}

\author{
Dezső Bednay* \\ Corvinus University of Budapest / PhD Program in Economic Theory \\ Fővám tér 8, Budapest, H-1093, Hungary \\ Email: bednay@gmail.com
}

July 10, 2013

\begin{abstract}
We consider von Neumann - Morgenstern stable sets in assignment games with one seller and many buyers. We prove that a set of imputations is a stable set if and only if it is the graph of a certain type of continuous and monotone function. This characterization enables us to interpret the standards of behavior encompassed by the various stable sets as possible outcomes of well-known auction procedures when groups of buyers may form bidder rings. We also show that the union of all stable sets can be described as the union of convex polytopes all of whose vertices are marginal contribution payoff vectors. Consequently, each stable set is contained in the Weber set. The Shapley value, however, typically falls outside the union of all stable sets.
\end{abstract}

\section{Introduction}

Assignment games (Shapley and Shubik, 1972) are models of two-sided matching markets with transferable utilities where the aim of each player on one side is to form a profitable coalition with a player on the other side. Since only such bilateral cooperations are worthy, these games are completely defined by the matrix containing the cooperative worths of all possible pairings of players from the two sides.

\footnotetext{
*Supported by OTKA K72856
} 
Shapley and Shubik (1972) showed that the core of an assignment game is precisely the set of dual optimal solutions to the assignment optimization problem on the underlying matrix of mixed-pair profits. This implies that (i) every assignment game has a non-empty core; (ii) the core can be determined without explicitly generating the entire coalitional function of the game; and (iii) there are two special vertices of the core, in each of which every player from one side of the market receives his/her highest core payoff while every player from the other side of the market receives his/her lowest core payoff.

Besides the above fundamental results concerning the core, several important contributions dealing with other solution concepts have been published in the last decade. The classical solution concept proposed and studied by von Neumann and Morgenstern (1944) in their monumental work has remained an intriguing exception, although Solymosi and Raghavan (2001) characterized a subclass of assignment games where the core is the unique stable set. The existence question in the general case was settled affirmatively by Núñez and Rafels (2009), who proved that, as conjectured by Shapley (cf. Section 8.4 in (Shubik, 1984)), the union of the cores of certain derived subgames is always a stable set.

In this paper ${ }^{1}$ we restrict our attention to the one-seller case that was already related to the classical auction setting with one seller and many buyers by Schotter (1974). In general, assignment games are useful models to study multi-item auction situations, see e.g. (Demange, Gale, Sotomayor, 1986).

The rest of the paper is as follows. The next section contains the necessary basic definitions and results. In Section 3 not just we show that any oneseller assignment game admits (typically infinite many) stable set solutions, but also give a characterization of how any stable set in these games must look like. Further results are presented in Section 4, where we give a precise description of the union of all stable sets as the union of convex polytopes all of whose vertices are marginal contribution payoff vectors, and conclude that each stable set is contained in the Weber set.

\footnotetext{
${ }^{1}$ This paper is based on Chapter 4 of the author's MSc thesis (Bednay, 2009) written at Corvinus University of Budapest under the supervision of Prof. Tamás Solymosi. The presentation has benefited from comments of participants at the SING-5 Conference in Amsterdam.
} 


\section{Preliminaries}

\subsection{Basic definitions}

A transferable utility cooperative game on the nonempty finite set $P$ of players is defined by a coalitional function $w: 2^{P} \rightarrow \mathbb{R}$ satisfying $w(\varnothing)=0$. The function $w$ specifies the worth of every coalition $S \subseteq P$.

Given a game $(P, w)$, a payoff allocation $x \in \mathbb{R}^{P}$ is called efficient, if $x(P)=w(P)$; individually rational, if $x_{i}=x(\{i\}) \geq w(\{i\})$ for all $i \in P$; coalitionally rational, if $x(S) \geq w(S)$ for all $S \subseteq P$; where, using the standard notation, $x(S)=\sum_{i \in S} x_{i}$ if $S \neq \varnothing$, and $x(\varnothing)=0$. We denote by $\mathcal{I}(P, w)$ the imputation set (i.e., the set of efficient and individually rational payoffs), and by $\mathcal{C}(P, w)$ the core (i.e., the set of efficient and coalitionally rational payoffs) of the game $(P, w)$.

The game $(P, w)$ is called superadditive, if $S \cap T=\varnothing$ implies $w(S \cup T) \geq$ $w(S)+w(T)$ for all $S, T \subseteq P$; balanced, if its core $\mathcal{C}(P, w)$ is not empty.

Given a game $(P, w)$, the excess $e(S, x):=w(S)-x(S)$ is the usual measure of gain (or loss if negative) to coalition $S \subseteq P$ if its members depart from allocation $x \in \mathbb{R}^{P}$ in order to form their own coalition. Note that $e(\varnothing, x)=0$ for all $x \in \mathbb{R}^{P}$, and that

$$
\mathcal{C}(P, w)=\left\{x \in \mathbb{R}^{P}: e(P, x)=0, e(S, x) \leq 0 \forall S \subset P\right\},
$$

i.e., the core is the set of allocations which yield nonpositive excess for all coalitions.

We say that allocation $y$ dominates allocation $x$ via coalition $S$ (notation: $\left.y \operatorname{dom}_{S} x\right)$ if $y(S) \leq w(S)$ and $y_{k}>x_{k} \forall k \in S$. We further say that allocation $y$ dominates allocation $x$ (notation: $y \operatorname{dom} x$ ) if there is a coalition $S$ such that $y$ dominates $x$ via $S$. Note that the dominance relation is irreflexive but need not be either asymmetric or transitive. This is the major source of the difficulties encountered when working with the following solution concept advocated by von Neumann and Morgenstern (1944). A (nonempty) set $Z$ of imputations is called a stable set if the following two conditions hold:

- (internal stability): there exist no $x, y \in Z$ such that $y \operatorname{dom} x$

- (external stability): for every $x \in \mathcal{I} \backslash Z$ there exists $y \in Z$ such that $y \operatorname{dom} x$ 
Note that the core is always a set of imputations which satisfies internal stability. It is commonly known that in superadditive games the core is precisely the set of imputations which are not dominated by any other imputation. Consequently, the core is a subset of any stable set.

Observe that for $x, y \in \mathcal{I}$, if $y \operatorname{dom}_{S} x$ then (i) $x(S)<w(S)$, i.e. an imputation can be dominated only via coalitions having positive excess at that imputation; and (ii) $2 \leq|S| \leq|P|-1$, i.e. among imputations domination can occur only via a proper coalition containing at least two players. Another useful observation is that inessential coalitions are redundant for the domination relation. We call coalition $S$ inessential in game $w$, if $w(S) \leq \sum_{1 \leq j \leq r} w\left(S_{j}\right)$ for a partition $S=\bigcup_{1 \leq j \leq r} S_{j}$, and call $S$ essential if it is not inessential. Suppose now that $y \operatorname{dom}_{S} x$ for some $S$ that is inessential because $w(S) \leq \sum_{1 \leq j \leq r} w\left(S_{j}\right)$ with a partition $S=\bigcup_{1 \leq j \leq r} S_{j}$. Then we must have $y \operatorname{dom}_{S_{j}} x$ for some $1 \leq j \leq r$. Consequently, if $\mathcal{E}(P, w)$ denotes the set of all essential coalitions in game $(P, w)$ then dom $=\bigcup_{S \in \mathcal{E}(P, w)} \operatorname{dom}_{S}$.

\subsection{One-seller assignment games}

In this paper we consider the following special type of assignment games. The player set is $P=\{0\} \cup N$, player 0 is called the seller, and players $j \in N=\{1, \ldots, n\}$ are called buyers. The coalitional function $w=w_{A}$ is generated from the $1 \times n$ nonnegative matrix $A=\left[\begin{array}{lll}a_{1} & a_{2} & \ldots\end{array} a_{n}\right]$ consisting of the profits the seller can make with the single buyers. We define

$$
w_{A}(S)= \begin{cases}\max _{j \in S \cap N} a_{j} & \text { if } 0 \in S, S \cap N \neq \varnothing \\ 0 & \text { else }\end{cases}
$$

Throughout we will assume that $a_{1} \geq a_{2} \geq \ldots \geq a_{n} \geq 0$, and call player 1 the strongest buyer, player 2 the second strongest buyer, and so on and so forth. Player $n$ is the weakest buyer. One-seller assignment games are obviously superadditive.

To emphasize the special role of the seller, we shall write the payoff allocations as $(u ; \boldsymbol{v}) \in \mathbb{R} \times \mathbb{R}^{n}$. Then the imputation set of one-seller assignment game $w_{A}$ is $\mathcal{I}\left(w_{A}\right)=\left\{(u ; \boldsymbol{v}) \geq(0 ; \mathbf{0}): u+\sum_{j \in N} v_{j}=a_{1}\right\}$. It is easily seen that the core is the line segment $\mathcal{C}\left(w_{A}\right)=\left\{(u ; \boldsymbol{v}) \in \mathcal{I}\left(w_{A}\right): a_{2} \leq u \leq\right.$ $\left.a_{1} ; v_{1}=a_{1}-u ; v_{j}=0(j=2, \ldots, n)\right\}$ with endpoints $\left(u=a_{1} ; \boldsymbol{v}=\mathbf{0}\right)$ and $\left(u=a_{2} ; v_{1}=a_{1}-a_{2}, v_{2}=\ldots=v_{n}=0\right)$, the seller-optimal and the buyer-optimal vertices, respectively. 
In one-seller assignment game $w_{A}$ domination among imputations is possible only via coalitions $\{0, j\}$ with $a_{j}>0$. Thus, we shall simply write $(u ; \boldsymbol{v}) \operatorname{dom}_{j}\left(u^{\prime} ; \boldsymbol{v}^{\prime}\right)$ if $u+v_{j} \leq a_{j}$ and $u>u^{\prime}, v_{j}>v_{j}^{\prime}$. Since the set of essential coalitions consists of these non-dummy mixed-pair coalitions and the single-player coalitions, but domination is not possible via the 0 -valued single-player coalitions, we clearly have

$$
\operatorname{dom}=\bigcup_{j \in N: a_{j}>0} \operatorname{dom}_{j}
$$

An important feature of one-seller assignment games is that its core is a line segment. However there are other assignment games that have a line segment core, for example the famous Böhm-Bawerk horse market game discussed by Shapley and Shubik (1972).

We think however that our characterization only holds for one-seller assignment games. In the simplest case of assignment games with more sellers, our characterization gives only sufficient but not necessary conditions. (cf. Section 8.4 in (Shubik, 1984)).

\section{The characterization}

We consider the one-seller assignment game $w_{A}$ induced by the nonnegative matrix

$$
A=\left[\begin{array}{llll}
a_{1} & a_{2} & \ldots & a_{n}
\end{array}\right]
$$

satisfying $a_{1} \geq a_{2} \geq \cdots \geq a_{n} \geq 0=a_{n+1}$. To simplify notation, we drop reference to $w_{A}$ or $A$ whenever this causes no confusion.

The following subset of imputations will be important

$$
X=\left\{(u, \boldsymbol{v}) \in \mathcal{I}: v_{i}=0 \text { or } u+v_{i} \leq a_{i}, \text { for all } i \in N\right\}
$$

or equivalently,

$$
X=\left\{(u, \boldsymbol{v}) \in \mathcal{I}: v_{i} \leq\left(a_{i}-u\right)_{+}, \text {for all } i \in N\right\}
$$

where $(x)_{+}:=\max \{x, 0\}$ denotes the positive part of real number $x$. At imputations in $X$ none of the buyers get 'too much'. More precisely, if a buyer gets a positive payoff then it cannot exceed what this buyer can achieve when paired with the seller. 
We say that a subset $Z$ of imputations is a " $\left[0, a_{1}\right]$-continuous, monotone curve in $X$ ", if there exists a continuous and componentwise monotone nonincreasing function $f:\left[0, a_{1}\right] \rightarrow \mathbb{R}^{n}$ such that $\operatorname{graph}(f)=\left\{\left(u, f_{1}(u), \ldots, f_{n}(u)\right)\right.$ : $\left.0 \leq u \leq a_{1}\right\}=Z \subseteq X$. Notice that any such $Z$ contains the extreme point $\left(u=a_{1}, \boldsymbol{v}=\mathbf{0}\right)$ of the imputation set (and of the core), and since $X \subseteq \mathcal{I}$ in every element of these curves the seller and the strongest buyer can dominate.

We denote by $\mathcal{I}(u)$ the set of imputations in which the seller's payoff equals $u$.

Theorem 3.1 Let $w_{A}$ be a one-seller assignment game. Then $Z \subseteq \mathcal{I}$ is a stable set in $w_{A}$, if and only if $Z$ is a $\left[0, a_{1}\right]$-continuous, monotone curve in $X$.

Proof.

Sufficiency: Let $Z$ be an "[0, $\left.a_{1}\right]$-continuous, monotone curve in $X$ ".

Internal stability of $Z$ comes from monotonicity, since for any two imputations $(u, \boldsymbol{v})$ and $\left(u^{\prime}, \boldsymbol{v}^{\prime}\right) \in Z$ with $u>u^{\prime}$ we have $\boldsymbol{v} \leq \boldsymbol{v}^{\prime}$.

To see external stability of $Z$, let $(u, v) \in \mathcal{I} \backslash Z$. Then $0 \leq u<a_{1}$, for otherwise $u \geq a_{1}$ would imply $\boldsymbol{v}=\mathbf{0}$, but then $(u, \boldsymbol{v})$ could not be an imputation outside of $Z$. We claim that there exists an imputation $\left(u^{\prime}, \boldsymbol{v}^{\prime}\right) \in$ $Z$ satisfying $u<u^{\prime}$ and $v_{i}<v_{i}^{\prime}$ for some $i \in N$. If not, then for every large enough $n$ the imputation $\left(u^{n}, \boldsymbol{v}^{n}\right)=\left(u+\frac{1}{n}, f\left(u+\frac{1}{n}\right)\right) \in Z$ would satisfy $f_{i}\left(u+\frac{1}{n}\right) \leq v_{i}$ for all $i \in N$. By continuity, $\lim _{n \rightarrow \infty}\left(u^{n}, \boldsymbol{v}^{n}\right)=(u, \boldsymbol{v}) \in Z$, is a contradiction. Thus, there exists an imputation $\left(u^{\prime}, \boldsymbol{v}^{\prime}\right) \in Z$ satisfying $u<u^{\prime}$ and $v_{i}<v_{i}^{\prime}$ for some $i \in N$, but since $Z \subseteq X$, we have $\left(u^{\prime}, \boldsymbol{v}^{\prime}\right) \operatorname{dom}_{i}(u, \boldsymbol{v})$.

Necessity: Let $Z$ be a stable set. We prove its properties in five steps.

(1) $Z \subseteq X$

Suppose not, and there exists $(u, \boldsymbol{v}) \in Z \backslash X$, meaning that there exist $(u, \boldsymbol{v}) \in Z$ and $i \in N$ such that $u+\sum_{j=1}^{n} v_{j}=a_{1}, v_{i}>0, u+v_{i}>a_{i}$, $u \geq 0, \boldsymbol{v} \geq \mathbf{0}$. Let $\epsilon=\min \left(v_{i}, u+v_{i}-a_{i}\right)$. Obviously, $\epsilon>0$. We define $u^{\prime}=u+\frac{\epsilon}{2}, v_{1}^{\prime}=v_{1}+\frac{\epsilon}{2}, v_{i}^{\prime}=v_{i}-\epsilon$, and $v_{k}^{\prime}=v_{k}$ for any other $k \neq 1, i$. Then $\left(u^{\prime}, \boldsymbol{v}^{\prime}\right) \in \mathcal{I}$, because its total payoff also equals $a_{1}$ and all payoffs are nonnegative (only $v_{i}^{\prime}<v_{i}$, but by the definition of $\epsilon$ it is still nonnegative). Since $\left(u^{\prime}, \boldsymbol{v}^{\prime}\right) \operatorname{dom}_{1}(u, \boldsymbol{v})$, internal stability of $Z$ implies $\left(u^{\prime}, \boldsymbol{v}^{\prime}\right) \notin Z$. Then, by external stability, there is a $\left(u^{\prime \prime}, \boldsymbol{v}^{\prime \prime}\right) \in Z$ for which $\left(u^{\prime \prime}, \boldsymbol{v}^{\prime \prime}\right) \operatorname{dom}\left(u^{\prime}, \boldsymbol{v}^{\prime}\right)$. This domination cannot happen via coalition $\{0, i\}$, because $u^{\prime}+v_{i}^{\prime}=u+v_{i}-\frac{\epsilon}{2}>$ $u+v_{i}-\epsilon \geq u+v_{i}-\left(u+v_{i}-a_{i}\right)=a_{i}$. But if $\left(u^{\prime \prime}, \boldsymbol{v}^{\prime \prime}\right) \operatorname{dom}_{j}\left(u^{\prime}, \boldsymbol{v}^{\prime}\right)$ then also $\left(u^{\prime \prime}, \boldsymbol{v}^{\prime \prime}\right) \operatorname{dom}_{j}(u, \boldsymbol{v})$ (because $u^{\prime \prime}>u^{\prime}>u$ and $v_{j}^{\prime \prime}>v_{j}^{\prime} \geq v_{j}$ for all $j \neq i$ ), a contradiction of internal stability of $Z$. 
(2) $Z$ is the graph of a function $f$

We claim that for any $u \in\left[0, a_{1}\right]$ there exists at most one imputation $(u, \boldsymbol{v}) \in Z$. Suppose not, and there exist $(u, \boldsymbol{v}),\left(u, \boldsymbol{v}^{\prime}\right) \in Z$ with $\boldsymbol{v} \neq \boldsymbol{v}^{\prime}$ and, w.l.o.g., $v_{1} \leq v_{1}^{\prime}$. Let $v_{i}^{\prime \prime}=\min \left(v_{i}, v_{i}^{\prime}\right)$ for all $i \in N$ and $\epsilon=\sum_{i \in N}\left(v_{i}-v_{i}^{\prime \prime}\right)$. Obviously, $\epsilon>0$. We define $\left(u^{1}, \boldsymbol{v}^{\mathbf{1}}\right)=\left(u+\frac{\epsilon}{2}, v_{1}+\frac{\epsilon}{2}, v_{2}^{\prime \prime}, \ldots, v_{n}^{\prime \prime}\right)$, that is clearly an imputation, implying in particular that $u^{1}+v_{1}^{1} \leq u^{1}+\sum_{j=1}^{n} v_{j}^{1}=a_{1}$. Then $\left(u^{1}, \boldsymbol{v}^{\mathbf{1}}\right) \operatorname{dom}_{1}(u, \boldsymbol{v})$, thus, by internal stability, $\left(u^{1}, \boldsymbol{v}^{\mathbf{1}}\right) \notin Z$. External stability implies that there exists $\left(u^{2}, \boldsymbol{v}^{\mathbf{2}}\right) \in Z$ and $i \in N$ for which $\left(u^{2}, \boldsymbol{v}^{\mathbf{2}}\right) \operatorname{dom}_{i}\left(u^{1}, \boldsymbol{v}^{\mathbf{1}}\right)$. But from $u^{2}>u^{1}>u$ and $v_{i}^{2}>v_{i}^{1} \geq \min \left(v_{i}, v_{i}^{\prime}\right)$ it follows that $\left(u^{2}, \boldsymbol{v}^{2}\right)$ dominates $(u, \boldsymbol{v})$ if $v_{i}^{\prime \prime}=v_{i}$ or it dominates $\left(u, \boldsymbol{v}^{\prime}\right)$ if $v_{i}^{\prime \prime}=v_{i}^{\prime}$, either way, a contradiction to internal stability. Therefore, from stable set $Z$ we can define a scalar-vector function $f$ by $f(u)=\boldsymbol{v}$ whenever $(u, \boldsymbol{v}) \in Z$.

(3) $f$ is componentwise monotone non-increasing

We claim that $(u, \boldsymbol{v}),\left(u^{\prime}, \boldsymbol{v}^{\prime}\right) \in Z$ and $u<u^{\prime}$ imply $\boldsymbol{v} \geq \boldsymbol{v}^{\prime}$. Suppose not, and there exists $j \in N$ such that $v_{j}<v_{j}^{\prime}$. By step (1), $Z \subseteq X$, thus $u^{\prime}+v_{j}^{\prime} \leq a_{j}$. Therefore, $\left(u^{\prime}, \boldsymbol{v}^{\prime}\right) \operatorname{dom}_{j}(u, \boldsymbol{v})$, a contradiction to internal stability.

(4) $f$ is defined for every $u \in\left[0, a_{1}\right]$

Since $Z$, like any stable set, contains the core, $f$ is defined for every $u \in$ $\left[a_{2}, a_{1}\right]$. Suppose that $\mathcal{I}(u) \cap Z=\varnothing$ for some $0 \leq u<a_{2}$, i.e. $f$ is not defined for $u$. Let $\bar{u}$ be the infimum of the (nonempty) set $\{h>u: \mathcal{I}(h) \cap Z \neq$ $\varnothing\}$. In step (2) we have already proved that if nonempty, $\mathcal{I}(h) \cap Z$ is a singleton, so any decreasing sequence $h \searrow \bar{u}$ with $\mathcal{I}(h) \cap Z \neq \varnothing$ induces a sequence $(h, f(h))$ in $Z$. Since $Z$, like any stable set, is closed, we get that $\mathcal{I}(\bar{u}) \cap Z \neq \varnothing$, i.e. there exists (by step (2), a unique) imputation $(\bar{u}, \underline{\boldsymbol{v}}) \in Z$. In step (3) we have already proved that every component of $f$ is monotone non-increasing, so for any $h>\bar{u}$, if defined, $f(h) \leq \underline{\boldsymbol{v}}$. It follows from our supposition that $\bar{u}>u$. Take $u^{\prime}=u$ and $v_{i}^{\prime}=\underline{v}_{i}+\frac{\bar{u}-u}{n}$ for all $i \in N$, that is obviously an imputation, but not in $Z$. The monotonicity of $f$ implies that $\left(u^{\prime}, \boldsymbol{v}^{\prime}\right)$ cannot be dominated by some $(h, f(h)) \in Z$ with $h \geq \bar{u}$. But external stability implies that $Z$ contains an imputation $\left(u^{\prime \prime}, \boldsymbol{v}^{\prime \prime}\right)$ that dominates $\left(u^{\prime}, \boldsymbol{v}^{\prime}\right)$. It follows that $u=u^{\prime}<u^{\prime \prime}<\bar{u}$, a contradiction to the definition of $\bar{u}$. Therefore, $f$ must also be defined for every $u \in\left[0, a_{2}\right]$.

(5) $f$ is continuous

Since every stable set is closed and $f$ is a function defined on the interval $\left[0 ; a_{n}\right]$ we get immediately the continuity of the function $f$. 


\section{Further results}

In the previous section we have seen that in a one-seller assignment game there are (typically) infinite many stable sets, and have characterized them individually as continuous, monotone curves in a certain subset $X$ of imputations. Now we will give a subset of imputations (smaller than $X$ ) such that all of its points are included in some stable set. We define

$$
U=\left\{(u, \boldsymbol{v}) \in \mathcal{I} \forall i: \sum_{j=i}^{n} v_{j} \leq\left(a_{i}-u\right)_{+}\right\} .
$$

This set is very similar to set $X$, but in $U$ not even 'tail-coalitions' of buyers can get 'too much'. More precisely, in $U$ we require that if the 'tail-coalition' of the weakest $n-i+1$ buyers get in total a positive payoff then it should be feasible for their coalition with the seller. Thus, we equivalently describe

$$
U=\left\{(u, \boldsymbol{v}) \in \mathcal{I} \forall i: v_{i}=\ldots=v_{n}=0 \text { or } u+\sum_{j=i}^{n} v_{j} \leq a_{i}\right\}
$$

Remark 4.1 $U \subseteq X$, because $\sum_{j=i}^{n} v_{j} \geq v_{i}$, so $\sum_{j=i}^{n} v_{j} \leq\left(a_{i}-u\right)_{+}$for all $i \in N$ implies $v_{i} \leq\left(a_{i}-u\right)_{+}$for all $i \in N$.

Remark $4.2 U$ can be a strict subset of $X$. Take, for example, $A=\left[\begin{array}{ccc}10 & 5 & 5\end{array}\right]$.

In the induced one-seller assignment game, imputation $(u, \boldsymbol{v})=(3 ; 3,2,2)$ is in $X$, but it is not in $U$, since the coalition of the last two buyers together with the seller gets in total more than what they could achieve: $u+v_{2}+v_{3}=$ $7 \not 5=a_{2}$.

Theorem 4.1 The union of all stable sets is precisely the set $U$.

ProOF.

First we prove $U \supseteq \bigcup_{Z \text { stable set }} Z$.

Suppose not, and there exists a stable set $Z$ and an imputation $(u, \boldsymbol{v}) \in Z$ such that $(u, \boldsymbol{v}) \in X \backslash U$. This means that $\sum_{j=k}^{n} v_{j}>\left(a_{k}-u\right)_{+}$for some buyer $k \in N$. Let $\left(u^{\prime}, \boldsymbol{v}^{\prime}\right)$ be that (unique) imputation in $Z$ for which the seller's payoff is $u^{\prime}=a_{k}$. Then, we must have $v_{k}^{\prime}=v_{k-1}^{\prime}=\ldots=v_{n}^{\prime}=0$, because of $\left(u^{\prime}, \boldsymbol{v}^{\prime}\right) \in X$.

There are two cases: 
1. If $u^{\prime}=a_{k}<u$, then by monotonicity, $\boldsymbol{v}^{\prime} \geq \boldsymbol{v} \geq \mathbf{0}$, implying $v_{k}=v_{k+1}=$ $v_{n}=0$, but this leads to the contradiction $0=\sum_{j=k}^{n} v_{j}>\left(a_{k}-u\right)_{+}=0$.

2. If $u^{\prime}=a_{k} \geq u$, then $\left(a_{k}-u\right)_{+}=a_{k}-u$, implying $\sum_{j=k}^{n} v_{j}>a_{k}-u$. This is again impossible, since $(u, \boldsymbol{v})$ and $(u, \boldsymbol{v})$ are imputations and by monotonicity, $u^{\prime}-u=\sum_{j=1}^{n}\left(v_{j}-v_{j}^{\prime}\right) \geq \sum_{j=k}^{n}\left(v_{j}-v_{j}^{\prime}\right)=\sum_{j=k}^{n} v_{j}>$ $\left(a_{k}-u\right)_{+}=u^{\prime}-u$.

Second we prove $U \subseteq \bigcup_{Z \text { stable set }} Z$.

Let $(u, \boldsymbol{v}) \in U$. We will exhibit a stable set $Z$ for which $(u, \boldsymbol{v}) \in Z$. Consider the following construction of a $\left(\boldsymbol{u}^{\prime} ; \boldsymbol{v}^{\prime}\right)$ stable curve:

1. If $u^{\prime}<u$, let $\boldsymbol{v}^{\prime}=\left(v_{1}+u-u^{\prime}, v_{2}, \ldots, v_{n}\right)$. Naturally, $\left(u^{\prime}, \boldsymbol{v}^{\prime}\right) \in X$ since $u^{\prime}+v_{i}^{\prime} \leq u+v_{i} \leq a_{i}$.

2. If $u^{\prime}>u$, then if $u^{\prime}-u=v_{n}+v_{n-1}+\ldots+v_{k+1}+x$, where $0<x \leq v_{k}$, let $\boldsymbol{v}^{\prime}=\left(v_{1}, v_{2}, \ldots, v_{k-1}, v_{k}-x, 0,0, \ldots, 0\right)$. We claim that $\left(u^{\prime}, \boldsymbol{v}^{\prime}\right) \in X$. If $v_{i}^{\prime}>0$ then $u^{\prime}+v_{i} \leq u+\sum_{j=i}^{n} v_{j} \leq a_{i}$, since $v_{i}^{\prime}>0$ implies $v_{i}>0$, that is $\sum_{j=i}^{n} v_{j}>0$, thus $\sum_{j=i}^{n} v_{j} \leq a_{i}-u$.

This procedure means that from a given imputation $(u, v)$ we construct a curve in the following way: any decrease in the seller's payoff $u$ goes to the strongest buyer, whereas, the increase in $u$ is taken first from the weakest buyer, then when payoff $v_{n}$ is exhausted, from the second weakest buyer, then when also payoff $v_{n-1}$ is exhausted, from the third weakest buyer, and so on, till only the strongest buyer's payoff $v_{1}$ can be decreased. The curve constructed this way is obviously continuous and monotone, and since it is in $X$, it is a stable set.

In the example in Remark 2 we can't construct a stable set through the payoff vector $(3 ; 3,2,2)$ because when we increase the sellers payoff by 1 we have to decrease the second and also the third buyers payoff by 1 to be in the set $V$ because the pair including the seller and the second (or the third) buyer can't get more than 5. But because of the monotonicity we can not do that.

We can offer the following interpretation for a stable set, if the game represents a single-item auction situation. If all buyers act competitively, the strongest buyer can outbid every other buyer, so he is left alone to negotiate with the seller, the outcome is in the core. The lowest price that can be achieved in such a competitive setting is determined by the valuation of the 
second strongest buyer, since below $a_{2}$ buyer 2 could also play an active role in the negotiations. The price can be lowered, however, if buyers 1 and 2 agree not to outbid each other, e.g. buyer 2 acts as if he were a dummy buyer. Forming a bidder ring, buyers 1 and 2 can, in principle, lower the selling price down to $a_{3}$, the price level when the third strongest buyer could enter the scene as an active bidder. If buyer 3 is also included in the ring, the selling price could be further lowered, etc..

Of course, the ring-members should agree on the distribution of the gain they could achieve, they should specify how buyer 1, who remains on the negotiation scene, will compensate the weaker buyers, who pretend to be dummies. The stable sets describe exactly the conceivable such compensation schemes. The function $p \mapsto f(p)$ specifies how the gain $a_{1}-p$ of the ring will be distributed, if no ring-member bids more than $p$ for the item. The curve must be in set $U$, because $\sum_{j=i}^{n} f_{j}(p)$ is the total compensation amount given by buyer 1 to buyers $i, \ldots, n$, but even without their consent and cooperation the strongest buyer could achieve the item at a price $a_{i}$. That explains the restriction $\sum_{j=i}^{n} f_{j}(p) \leq\left(a_{i}-p\right)_{+}$. Forms of such compensation schemes are discussed by Schotter (1974) for various well-known auction mechanisms.

Let us now see in two examples how the stable sets look like.

First, a market with one seller and two buyers, a situation that has been thoroughly discussed already by von Neumann and Morgenstern (1953, section 62). We take in particular the profit matrix $A=\left[\begin{array}{ll}100 & 75\end{array}\right]$.

The set of imputations is the whole triangle in Figure 1. The core is the line segment between the points $(100 ; 0,0)$ and $(75 ; 25,0)$. Set $U$ is the union of the core and the grey triangle with corners $(75 ; 25,0),(0 ; 100,0)$, $(0 ; 25,75)$. The thick curve is a stable set. The striped angle-regions indicate the set of imputations dominated by the given imputation. As von Neumann and Morgenstern (1953, p. 572) have already concluded a stable set should contain "the upper part, corresponding to the competition of the two buyers" (i.e. the core), and "a lower part, corresponding to a coalition of the two buyers against the seller". This lower part is a curve going from the lowest point of the upper part down to a point on the bottom side of the imputation triangle "the direction of which never deviates from the vertical by more than $30^{\circ}$ " (p. 412), "hence it is continuous" (footnote 1, p. 412).

In our second example, there are 3 buyers, and the profit matrix is $A=$ $\left[\begin{array}{lll}100 & 75 & 40\end{array}\right]$ 
Figure 1:

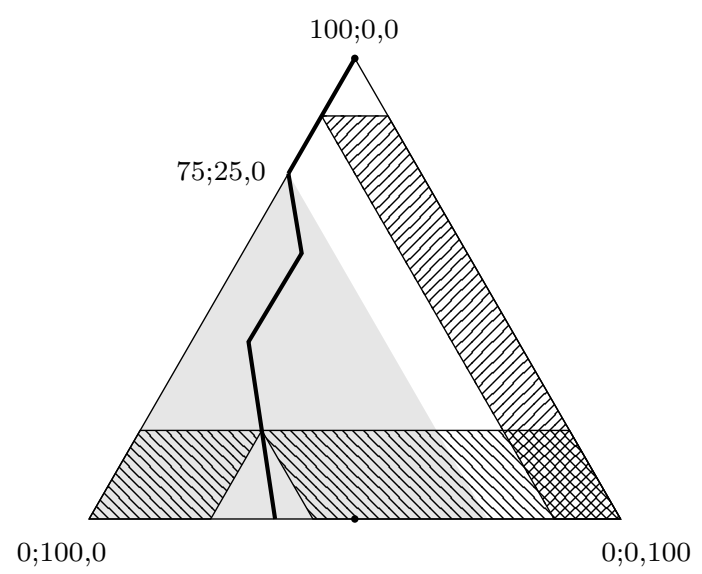

The imputation set is the whole tetrahedron in Figure 2. The core is the line segment between the points $(100 ; 0,0,0)$ and $(75 ; 25,0,0)$. Set $U$ here can be described as the union of three convex polytopes: the first is the core, i.e. the line segment on the $v_{2}=v_{3}=0$ edge of the imputation set with vertices $(100 ; 0,0,0)$ and $(75 ; 25,0,0)$; the second is the triangle in the $v_{3}=0$ side of the imputation set with vertices $(75 ; 25,0,0),(40 ; 60,0,0),(40 ; 25,35,0)$; the third is the roof-like polytope on the $u=0$ base of the imputation set with vertices $(0 ; 100,0,0),(40 ; 60,0,0),(0 ; 60,0,40),(40 ; 25,35,0),(0 ; 25,35,40)$, $(0 ; 25,75,0)$.

In general, we can get the vertices of the set $U$ in the following way. If $a_{k} \geq u \geq a_{k+1}$ then since $\sum_{j=k+1}^{n} v_{j} \leq\left(a_{k+1}-u\right)_{+}=0$ we have $v_{k+1}=v_{k+2}=$ $\cdots=v_{n}$. In this case we can write the other inequalities in the following way for all $1 \leq i \leq k:\left(\sum_{j=i}^{n} v_{j}=\right) \sum_{j=i}^{k} v_{j} \leq a_{i}-u\left(=\left(a_{i}-u\right)_{+}\right)$. It can be easily seen that at the extremal points of these polytopes the seller gets $a_{l}$, the first buyer gets $a_{1}-a_{i_{1}}$, the second buyer gets $a_{i_{1}}-a_{i_{2}}, \ldots$, the l-th buyer gets $a_{i_{l-1}}-a_{i_{l}}$ for some $i_{1}, i_{2}, \ldots i_{l}=l$ monotone increasing positive integer sequence and $l=k$ or $l=k+1$. 
Figure 2:

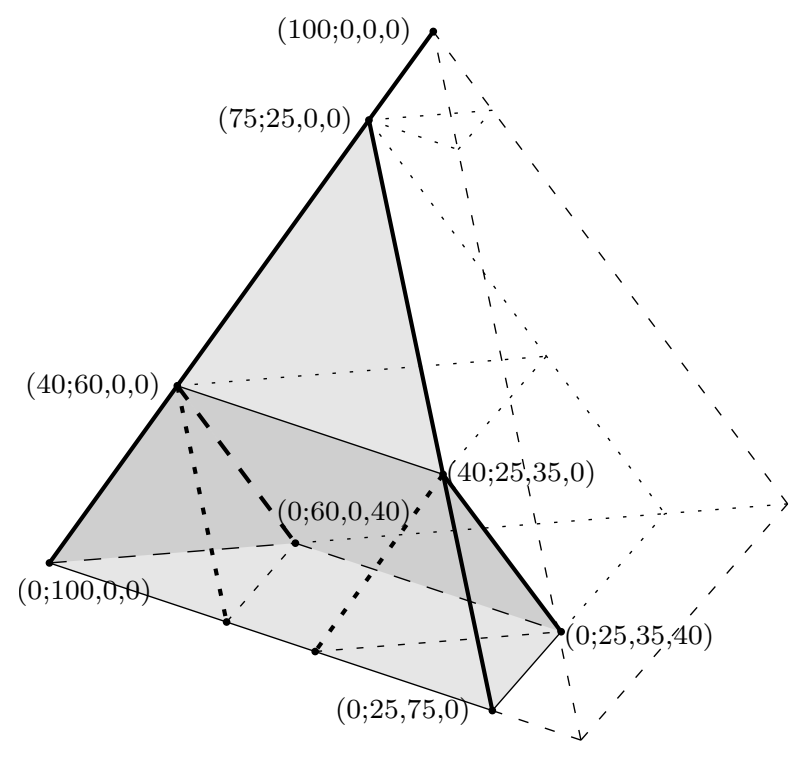

In order to describe more precisely these special imputations, we recall the concept of marginal contribution vectors (specialized to our setting). Let $\theta: P \rightarrow\{0,1, \ldots, n\}$ be an ordering of the players, i.e. the position of player $k$ is $\theta(k)$, whereas $\theta^{-1}(k)$ tells us which player occupies the $k$-th position in the ordering. Let $\Theta_{P}$ denote the set of all orderings of the player set $P$. In a given ordering $\theta \in \Theta_{P}$ the set of players before player $k$ is denoted by $P_{k}^{\theta}=\{j \in P \mid \theta(j)<\theta(k)\}$. Given a (one-seller assignment) game $w$ and an ordering $\theta \in \Theta_{P}$, we define the $(1+n)$-dimensional vector of marginal payoffs

$$
\boldsymbol{x}^{\theta}(w)=\left(x_{k}^{\theta}(w)=w\left(P_{k}^{\theta} \cup\{k\}\right)-w\left(P_{k}^{\theta}\right)\right)_{k \in P} .
$$

We will use the following sets of marginal payoff vectors: $M=\left\{\boldsymbol{x}^{\theta}(w)\right.$ : $\left.\theta \in \Theta_{P}\right\}$ and for $i=1, \ldots, n+1, M_{i}=\left\{(u, \boldsymbol{v}) \in M: u=a_{i}\right\}$ (recall that $\left.a_{n+1}=0\right)$. Clearly,

$$
M=\bigcup_{i=1}^{n+1} M_{i}
$$

Proposition 4.1 The vertices of set $U$ are marginal contribution vectors.

The $\left(a_{l} ; a_{1}-a_{i_{1}}, a_{i_{1}}-a_{i_{2}}, \ldots a_{i_{l-1}}-a_{i_{l}}, 0,0, \ldots, 0\right)$ vertex belongs to the following ordering: in the first $n-l+1$ place there comes the $n-l+1$ 
weakest buyer. After them comes the seller and gets $a_{l}$. Then comes the weakest buyer from the rest of the buyers with positive payoff, then the second weakest with positive payoff ....after the strongest buyer who has positive payoff come all the other buyers in some order. It is easy to see that these ordering gives us the marginal contribution vector what we needed.

\section{Theorem 4.2}

$$
U=\bigcup_{i=1}^{n} \operatorname{conv}\left(M_{i} \cup M_{i+1}\right)
$$

Let $U_{i}=\left\{(u, \boldsymbol{v}) \in U: a_{i} \geq u \geq a_{i+1}\right\}$. Obviously, $U=\bigcup_{i=1}^{n} U_{i}$. We will show that $U_{i}=\operatorname{conv}\left(M_{i} \cup M_{i+1}\right)$. Since for every $1 \leq i \leq n$ the set $U_{i}$ is a polyhedron and the vertices of $U_{i}$ are marginal contribution vectors, it is enough to see that $M \subseteq U$. Suppose not, and there exists an ordering $\theta$ of the player set and an index $j$ such that $\boldsymbol{x}^{\theta}=(u, \boldsymbol{v})$ and $\sum_{i=j}^{n} v_{i}>\left(a_{j}-u\right)_{+}$. There are two cases

1. If $u>a_{j}$, then one of the buyers stronger than the $j$-th comes before the seller, implying $v_{j}=v_{j+1}=\ldots=v_{n}=0$, but this leads to the contradiction $\sum_{i=j}^{n} v_{i}=0 \leq\left(a_{j}-u\right)_{+}$.

2. If $u \leq a_{j}$, then when the last buyer not stronger than the $j$-th one with a positive marginal contribution comes, the excess of the coalition $\{0, j, j+1, \ldots, n\}$ still nonpositive, and since the rest of the buyers from this coalition has 0 payoff $\sum_{i=j}^{n} v_{i}+u \leq a_{j}$, implying the contradiction $\sum_{i=j}^{n} v_{i} \leq a_{j}-u=\left(a_{j}-u\right)_{+}$.

We immediately get the following consequences.

Corollary 4.1 The convex hull of set $U$ coincides with the convex hull of all marginal vectors (i.e. the Weber set of the game).

Recall that in any cooperative game, the core is a subset of the Weber set. In one-seller assignment games we have the following stronger result.

Corollary 4.2 In one-seller assignment games, every stable set is contained in the Weber set.

The Shapley value is the best-known single-valued solution concept for cooperative games. It is the simple average of all marginal payoff vectors, hence it is a kind of center of the Weber set. In light of the previous two corollaries, it is natural to ask whether the Shapley value is always contained in a stable set. The following example gives a negative answer. 
Example 4.1 In the one-seller assignment game induced by profit matrix $A=\left[\begin{array}{ll}2 & 1\end{array}\right]$, no stable set contains the Shapley value.

Indeed, the $(1+2) !=6$ marginal payoff vectors are: $(0,2,0),(0,1,1)$, $(2,0,0)$ (3-times), and $(1,1,0)$. Thus, the Shapley value is $\left(\frac{7}{6}, \frac{4}{6}, \frac{1}{6}\right)$. This imputation, however, is not in set $U$, because $\left(a_{2}-\frac{7}{6}\right)_{+}=0<\frac{1}{6}$.

\section{References}

[1] D. Bednay, Stabil halmazok hozzárendelési játékokban (Stable sets in assignment games), Master's thesis, Corvinus University of Budapest, Budapest, (2009).

Online available: http://szd.lib.uni-corvinus.hu/1981/

[2] G. Demange, D. Gale, and M. Sotomayor, Multi-item auctions, The Journal of Political Economy, vol. 94, 863-872 (1986)

[3] M. Núñez and C. Rafels, Von Neumann-Morgenstern solutions in the assignment market Journal of Economic Theory, vol. 148, 1282-1291 (2013)

[4] A. Schotter, Auctioning Böhm-Bawerk's Horses, International Journal of Game Theory, vol. 3, 195-215 (1974)

[5] L. S. Shapley and M. Shubik, The assignment game I: The core International Journal of Game Theory, vol. 1, 111-130 (1972)

[6] M. Shubik, A Game-Theoretic Approach to Political Economy, Game Theory in the Social Sciences, vol. 2, (1984)

[7] T. Solymosi and T. Raghavan, Assignment games with stable core, International Journal of Game Theory, vol. 30, 177-185 (2001)

[8] J. von Neumann and O. Morgenstern, Theory of Games and Economic Behavior (Third edition), Princeton University Press, Princeton New Jersey, (1953) 